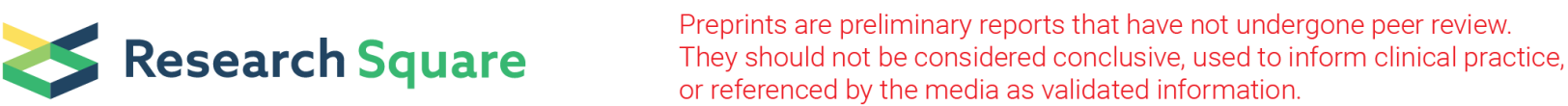

\section{Healthcare professionals' experiences of assessing, treating and preventing constipation among older patients during hospitalization: An interview study}

Veronica Lundberg ( $\nabla$ veronica.lundberg@ki.se)

Karolinska Institutet https://orcid.org/0000-0003-0899-5244

Anne-Marie Boström RN

Karolinska Institutet

Kristina Gottberg

Karolinska Institutet

Hanne Konradsen

Kobenhavns Universitet

Research article

Keywords: bowel, constipation, healthcare professionals, management, older patients

Posted Date: June 11th, 2020

DOl: https://doi.org/10.21203/rs.3.rs-33025/v1

License: (c) (1) This work is licensed under a Creative Commons Attribution 4.0 International License.

Read Full License

Version of Record: A version of this preprint was published at Journal of Multidisciplinary Healthcare on November 1st, 2020. See the published version at https://doi.org/10.2147/JMDH.S277727. 


\section{Abstract \\ Background}

Constipation is a common and troublesome condition among older patients and can result in a variety of negative health consequences. It is often undiagnosed or undertreated. Healthcare professionals have a responsibility to understand and address patients' overall healthcare needs so exploring their experiences is, therefore, highly relevant. The purpose of the study was to explore healthcare professionals' experiences of assessing, treating and preventing constipation among older patients.

\section{Methods}

A qualitative design with an exploratory approach was used. The participants were purposively sampled from three wards in a geriatric department in a medium-sized hospital in Sweden. Data were collected through focus group interviews and individual interviews and analyzed using content analysis.

\section{Results}

Three categories were generated: depending on resources and uncertainties, using different strategies, and approaching the patients' needs. In the care of older patients at risk of or with constipation, decisions were made based on personal knowledge, personal experience and clinical reasoning. A person-centered approach was highlighted but was not always possible to incorporate.

\section{Conclusions}

Different strategies for preventing and treating constipation were believed to be important, as was personcentered care, but were found to be difficult to perform. Healthcare professionals need adequate resources. Evidence-based clinical guidelines might help the delivery of high-quality care. Constipation needs to be given more focus in hospitals in order to potentially improve the care and patient outcomes for older people.

\section{Background}

Constipation is a common and troublesome condition among the general population (1). The prevalence is high in western countries (2), including Sweden (3), and may affect between $2-27 \%$ of people (4). In older people, the prevalence can vary between $50-80 \%$ depending on the healthcare setting (5). Constipation is associated with rectal pain and discomfort, affects overall well-being and can have a negative impact on quality of life (6). It can lead to consequences such as urinary retention, fecal incontinence, rectal prolapse, hemorrhoids, anal fissure and cardiovascular changes (7). 
Diagnosing constipation is often a challenge since there is no uniform definition of the criteria for diagnosis. Healthcare professionals, such as physicians and registered nurses, consider uncontrolled leakage of stool after bowel movement and the need for manual evacuation to be the most troublesome symptoms for patients (8) and tend to define constipation as less than three bowel movements per week (9). The Rome IV criteria for constipation focus on various symptoms, e.g., straining, lumpy or hard stools, sensation of incomplete evacuation, and sensation of anorectal obstruction; at least two symptoms must have been present for at least three months (10). Using these criteria could, however, be a challenge in clinical practice since they require the patient to recall their bowel pattern over the previous three months (11).

A large proportion of people who say they are constipated do not indicate many of the symptoms that physicians most often use to define constipation (12). The patients' definition is based on individual experiences with discomfort, hard stools, straining, infrequent bowel movements and abdominal pain being the most important symptoms (8). Constipation among older people living with cognitive impairment or dementia is common, but can be difficult to identify due to expressions of non-specific symptoms such as an aggressive behavior (13). Older people experience constipation as a painful challenge due to feelings of fatigue, loneliness, isolation and limitations on their social life $(14,15)$. The topic of constipation is associated with shame and taboo so the patient may be reluctant to talk about it and seek professional help (16-18). Research has shown that patients experience that healthcare professionals may not understand the severity of the situation (16). The problem also affects the family and society due to the increased healthcare costs incurred by the need for nursing time, emergency visits, hospital admissions and medication costs (19).

Many older patients are often exposed to strong pain-relieving drugs such as opioids and they are therefore increasingly affected by constipation. They are frequently recommended to adopt lifestyle changes such as higher intakes of fiber and fluid and increased physical activity $(20,21)$. However, these recommendations have limited evidence and have shown conflicting results $(20,22)$. The treatment of constipation is often traditional and based on clinical experience, theoretical considerations and data from the younger adult population (23).

In accordance with Swedish legislation, licensed healthcare professionals, such as registered nurses (RN) and physicians, have the primary responsibility for ensuring the provision of evidence-based and patientsafe care of high quality (24). Based on their knowledge, registered nurses assess the patients' situation and initiate and follow up on nursing actions so that patients receive the care they are entitled to. Senior Alert is a national Swedish quality register and a tool for the provision of systematic preventive care. It is used by registered nurses to conduct mandatory risk assessments regarding pressure ulcers, malnutrition, falls and oral health in persons receiving municipal or hospital care in order to improve the quality of the care (24). However, there is no risk assessment regarding bowel health in this register.

Healthcare professionals' knowledge about constipation in older patients, and its consequences and negative impact on quality of life, is low (25). The management of constipation is often a complex task 
due to multiple symptoms and their effects; this highlights the importance of examining healthcare professionals' views regarding the management of constipation. The aim of this study was to explore healthcare professionals' experiences of assessing, treating and preventing constipation in older patients.

\section{Methods}

\section{Design}

The study had an explorative study design with a qualitative approach (26). We collected data through a combination of focus groups and individual interviews. This combination has been previously described and is appropriate when the participants share a common frame of reference (27).

\section{Settings and participants}

We conducted the study on three different wards at the department of geriatric medicine in a mediumsized hospital in Sweden. The hospital consists of six wards, has 130 beds and is specialized in providing care to older patients who need medical treatment and rehabilitation. It employs 150 healthcare professionals and the average length of hospital stay is nine days.

A purposeful sampling method was used in order to recruit participants with a variety of experience in the care of older patients. The inclusion criteria were the following: registered nurse or physician with a minimum of one years' experience of caring for older people. These professions are those most involved in decision-making regarding clinical assessment and treatment. We contacted the ward managers, who sent the invitation to participate in the study to all healthcare professionals working on the wards. Persons who reported interest received an information letter about the study. We then contacted them regarding a time and place for the interview. In total, 20 healthcare professionals participated in this study, two focus groups with 12 participants and eight individual interviews. The participants represented a variety in terms of gender, age, education level and work experience (Table 1).

\section{Data collection}

A semi-structured interview guide was used during the focus groups and individual interviews. The interview guide was developed by the research group and reflected the study's objectives. Questions concerned their experiences of assessing, preventing and treating constipation, for example "Can you describe how you determine if a patient is at risk of developing constipation" and "Please tell me what you think and do when a patient is constipated?". We used subsequent probing and follow-up questions, such as "please explain what you mean" and "tell me more about this".

A moderator led the focus groups and an observer was present to ensure that all participants were given the opportunity to speak and share their experiences (28). The focus group interviews were conducted in November 2017 and each lasted 45-60 minutes. After each focus group interview, the moderator (VL) and the observer (HK) discussed the interview. The moderator is a gerontological nurse specialist and the observer is a nurse with experience of conducting focus group interviews. We offered individual 
interviews to participants who were not able to attend a focus group. The individual interviews were conducted in December 2017 and lasted for an average of 21 minutes (17-36 minutes). The participants chose the location of the interview at their workplace. Data collection continued until rich data were achieved.

\section{Data Analysis}

All interviews were audio-recorded and the first author transcribed the data verbatim. We used qualitative content analysis to analyze the data $(29,30)$. Initially, we read the transcribed material several times to gain a sense of the whole and to reflect upon the content. After reading the transcribed texts, we perceived that the "sense of the whole" was equivalent in the focus groups and individual interviews, so a combined data analysis was performed. We then identified meaning units that reflected the purpose of the study and condensed them, while retaining the core meaning. The condensed meaning units were then abstracted and a process of coding followed. Codes that have similar content and contexts were grouped, named and formed into sub-categories. Finally, we extracted the sub-categories and compared and combined those that were similar, thereby revealing categories $(29,30)$. Table 2 shows an example of the analysis process. The analysis process was reflective, moving back and forth between the different steps (29). The reflective process was reinforced by involving other researchers (HK and KG) who discussed, compared and reflected upon the results in order to reach consensus about the final categories. The trustworthiness of the analyzed material was strengthened by these discussions in the research team (31). Quotations are presented to illustrate the findings, which promotes confirmability (32, 33).

\section{Results}

The results from this study comprised three categories describing healthcare professionals' experiences of assessing, treating and preventing constipation. These categories were: depending on resources and uncertainties, using different strategies and approaching the patients' needs.

\section{Depending on resources and uncertainties}

\section{Depending on resources}

The participants experienced that they did not have enough time to assess patients' bowel function while taking care of the person's underlying healthcare situation that had led to them being admitted to hospital. Although there was an awareness among the participants about the risk of constipation, they reported that the workload contributed to constipation among the patients possibly being overlooked. The participants described several organizational factors that prevented them from prioritizing treatment and prevention due to time being needed for other tasks such as medication and documentation.

If we have a lot to do, this thing-elimination-may unfortunately come further down on the to-do-list (Participant 13) 
The participants described how they wanted to do their utmost for the patients but, as with assessment, prevention was also dependent on the time and availability of staff to, for example, increase patients' physical activity.

They are not physically active enough, if they could walk more, it (constipation) would have been a minor problem, but that's because of staff shortages (Participant 19)

Time restrictions also meant that the important dialogue with the patient about prevention of constipation was not prioritized and the patient was, therefore, not given the opportunity to be involved in the care. The participants also reported that lack of time made it difficult to evaluate if interventions to ensure regular bowel function were helping. The lack of assessment tools was also mentioned by the participants and they expressed uncertainty about whether the assessment of bowel function was mandatory in clinical practice or not.

\section{Professional uncertainties}

The participants described that they were lacking guidelines, which contributed to the use of different methods of assessing bowel function, difficulty assessing when patients needed preventive action, and uncertainty about what was considered to be adequate bowel movements. Despite this, the participants reported that they should motivate and provide information to the patients, while respecting their autonomy. For patients who were constipated, there were divergent opinions about different interventions used to treat constipation. The methods described were: sitting on the toilet instead of using a bedpan, contacting a dietitian for advice about diet, ensuring that the patient drinks enough fluids, manual evacuation and scheduled laxation. However, the participants expressed dislike for some of these methods, which they considered intrusive and a threat to the patients' integrity.

There was also uncertainty among healthcare professionals as to whether the interventions they used were evidence based or not. They relied, therefore, on their own experience and used interventions that they thought worked well. One participant questioned the routinely used practice of giving the same laxatives to everyone.

I don't even have any knowledge about the evidence and we don't have any routines, but otherwise it is usually these regulating agents (Participant 14)

Although issues related to constipation were considered important by the participants, they were often not documented in the patient records.

\section{Using different strategies}

\section{Individual strategies}

The participants expressed an uncertainty about what a patient's normal bowel pattern should be and they therefore used their own measures for the assessment and diagnosis of constipation. This could, for example, be counting the days between bowel movements and making a judgement based on that. 
maybe three days without having a bowel movement, then you start to get worried and try to discuss with the patient if it is normal for them (Participant 14)

The way that patients' bowel function was assessed varied, but the participants agreed that it was difficult to assess if a patient was constipated. The participants spoke about various strategies to prevent constipation. There was a general consensus about the importance of mobilization as this was timesaving in terms of prevention. The participants agreed that it was important to support and help the patients manage the situation by creating a stress-free and clean bathroom environment.

\section{Team strategies}

The healthcare professionals experienced that different methods were used in order to help keep the issue of constipation on the agenda. One strategy was discussing the patients' bowel function on the daily rounds with all staff members in the team.

We have morning rounds with all the people in the team and one point on the agenda is if the patient is constipated. We usually address this daily for all patients (Participant 17)

The participants suggested that this contributed to increased attention by all healthcare professionals and different treatment options could be discussed based on the patient's needs.

Another strategy was using an elimination list for every patient. This was a strategy preferred by some, whereas others believed that the information on such lists could often not be trusted. Instead, they thought that everyone in the team should monitor whether the patient had a bowel movement. The list was used as a daily "To- Do-List" where they read the plan for the patient during the hospital stay and wrote, for example, "Bowel Observation" on the list. However, this was also not considered by all to be a reliable method.

The healthcare professionals also described a strategy of keeping track of the patient's daily intake. They reported that when a patient might not want to eat and drink this could possibly be due to constipation.

\section{Pharmacological treatment}

The participants experienced that the most frequently used intervention for treating and preventing constipation was pharmacological laxatives. They described how they chose pharmacological treatment rather than trying non-pharmacological options. This choice was related to participants experiencing a lack of non-pharmacological treatment options on the ward. They also described difficulties in using nonpharmacological treatment among the older patients.

Patients'should not drink too little, have regular meals with extra fruit and vegetables, but we don't think about this when they come to hospital and nothing is normal (Participant 14)

They also described the importance of giving pharmacological treatment in parallel with, for example, analgesics. 
Surely, if she (the patient) has been described pain relief, then she must have parallel treatment with some preventive medication (Focus group 1)

The healthcare professionals experienced that it would be preferable if the choice of laxatives was made in consultation with the patient, but that this was not always possible. Instead, they described that they, as individual healthcare professionals, often decided which treatment should be initiated. They also described how treatment was often based on tradition and with respect to the patients' medical conditions, which determined the choice of pharmacological treatment.

\section{Approaching the patients' needs}

\section{Healthcare professionals' clinical reasoning}

The healthcare professionals' experienced that several factors played an important role in the management of constipation. These factors were related to both healthcare professionals and patients. Bowel health issues were experienced as challenging when assessing a patient with memory decline. In relation to these patients, the healthcare professionals described how they had to rely on their professional experience and ability to interpret patients' body language and "read between the lines". Objective assessments of the patients' feces, for example, were considered important.

One must learn, and be able to judge different feces, what they look like (Focus group 1)

A need for a team-based approach was highlighted. However, not everyone in the team was considered to have adequate competence and they used their own knowledge to support less educated staff in the management of constipation. Problematic situations could arise when the documentation was inadequate.

if you have received an insufficient report, then it is difficult, and, you must know what the consequences will be if someone doesn't have bowel movements (participant 13)

In these situations, they emphasized the importance of their own reasoning to determine whether a patient is at risk of constipation or not.

\section{Awareness of risk factors}

The participants highlighted the necessity of being aware of the risk factors, such as patients taking certain medications and being bedridden. These were considered important in relation to which treatment regimen to choose. Many patients with pain might not be able to cope and may experience fear of mobilizing. The participants, therefore, believed that this might also contribute negatively to the patient's ability to have regular bowel movements.

People who have fractures that make it difficult for them to move, maybe being afraid that it will hurt, then they usually get constipated. Pain makes them afraid to move as much as they really should and could (Focus group 2) 
The participants described this awareness as a skill that they had developed over the years, the longer they had worked.

In addition, when some patients with identified risks of becoming constipated refused treatment, the healthcare professionals reported that those with more experience tried to be flexible and find something that worked for the individual patient.

I've learned from experience to start with getting the patient to drink enough fluids (Participant 13)

The healthcare professionals also described other risk situations such as nausea and loss of appetite. Some participants argued, therefore, that it was important to be aware of each patient's nutritional status and understand when appropriate action should be initiated.

\section{A person-centered approach}

The healthcare professionals believed the management of constipation should be based on a personcentered approach. This involves talking to the patient and responding by trying to understand their individual situation.

So we ask the patient, how is it with your bowels today, are they working or not, then we assess this as well as what needs the patient has (Participant 17).

The healthcare professionals also assessed the patients' clinical abdominal status to form an idea of the patient's need for bowel movement.

Feeling the stomach, and listening to the stomach, with some you can feel that they are really constipated, but also if they are in pain (Participant 20).

According to the healthcare professionals, it was important to be aware that some patients are too focused on their bowels and therefore at risk of being overdosed with laxatives. The participants described an awareness and responsibility for providing care but at the same time difficulty in dealing with specific conditions when traditional treatments were not appropriate. Other complex situations were described where the patient's medical conditions influenced the assessment and choice of actions regarding constipation.

If they (patients) are severely ill with COPD and have breathlessness, they must not become severely constipated, they cannot push, nor should they have too frequent bowel movements with loose stools, because they can't hurry to the toilet (Participant 14)

It was considered important to be aware of the patient's individual symptoms. If, for example, a patient shouted spontaneously this could indicate that they were constipated. They also pointed out that older patients lack energy, which is important to consider when choosing actions. Hence, non-pharmacological interventions were not always considered appropriate for preventive purposes for each individual patient. 
Even though a person-centered approach was applied, healthcare professionals experienced difficulties. They implied that patients' individual experiences of care could be perceived differently depending on the patients' attitudes toward constipation, which could then affect the patient outcome.

\section{Discussion}

To our knowledge, this is the first study in Sweden illuminating healthcare professionals' experiences of assessing, treating and preventing constipation among older patients. Bowel health was considered an important part of the care of patients, but at the same time there was a lack of consensus on how to adequately assess, treat and prevent constipation.

Surprisingly, one of the key findings was that healthcare professionals experienced obstacles in the organization and relied on their own individual perceptions and attitudes, stating that they lacked an assessment tool. Saga, Seim, Morkved, Norton and Vinsnes (34) found that healthcare professionals often described difficulties in observing and identifying constipation, which also was found in this study. This may explain why healthcare professionals sometimes differ in their assessments and the limited utilization of assessment tools has been described $(35,36)$. The use of a constipation assessment scale has been demonstrated to be valuable in the assessment of constipation in nursing care and in different clinical settings (37). Improvement in evidence-based practice can be achieved through educating healthcare professionals in the use of assessment tools, standards and protocols as well as continuous follow up cycles to sustain their performance $(36,38)$.

The healthcare professionals emphasized the importance of clinical reasoning. Being able to interpret the patients' symptoms and body language, and understand what the problem is, was highlighted by the participants and played an important role in the management of constipation. The importance of clinical reasoning is emphasized by Woolley (39), who considered that healthcare professionals need to develop specific skills in order to be able to provide an accurate diagnosis. Muller-Staub, de Graaf-Waar (40) described the usefulness of the nursing process as a systematic approach to the organization of nursing knowledge and clinical decision-making through assessing, diagnosing and identifying appropriate interventions, as well as following up and evaluating the effects of these interventions.

Creating opportunities for healthcare professionals to communicate with patients about bowel issues may be one way to increase awareness about patients' subjective perceptions of constipation. This will open up for a dialogue between patients and healthcare professionals and might change clinical practice $(21,41,42)$. Several healthcare professionals described the need to communicate with the patient about experiences of bowel function; however, it was healthcare professionals who decided which treatment options should be initiated. A person-centered approach where patient's needs and subjective experience are taken into account has demonstrated better patient outcomes (43). To enable this, it is required that a person-centered approach is founded in the institutional leadership (44).

Another key finding of the present study was the different strategies that the participants reported for treating constipation. They described the importance of adhering to individual strategies as well as 
general strategies used on the ward, but they stated that pharmacological strategies were most commonly used and enabled individualized care. However, there seemed to be no consensus among the healthcare professionals' choice of strategies and they also expressed uncertainty as to whether treatment was evidence based or not and thereby used their own individual strategies. This uncertainty was also found in previous studies $(35,45)$.

Day, Wills and Coffey (46) argue that individualized care is recommended and that a combination of several treatment options may be needed to treat constipation and, above all, they emphasize investigating which strategies the patients themselves use. Dimidi, Cox, Grant, Scott and Whelan (47) argue that numerous symptoms are identified by patients but are often not diagnosed by healthcare professionals according to diagnostic criteria or tools. This indicates that it is necessary to take the patient's view into consideration when diagnosing the condition. The participants in this study also stated that the treatment should be individualized. However, they nevertheless proceeded to use pharmacological treatment as a first choice in a standardized manner and most of their actions were based on experience through clinical practice, which has also been described by others (48). It is not uncommon for healthcare professionals to rely on proven experience rather than evidence-based methods (49).

The healthcare professionals indicated that they were highly motivated to work with constipation in a preventive way, but were reliant on organizational resources, such as time and availability of staff, in order to meet the patients' individual needs. Having enough knowledge and time to assess and prioritize the topic of constipation was also found to be important in a study by Saga, Seim, Morkved, Norton and Vinsnes (34) and might be related to organizational prioritization $(41,50)$. In the interviews, the healthcare professionals reported that it was important to be aware of patients' risk factors and the consequences that constipation can lead to. A study by Goodman, Low and Wilkinson (51) previously found that healthcare professionals are aware of the importance of working preventively to avoid constipation. Such an awareness also emerged in this study through the participants' descriptions of their ambitions to prevent constipation. However, despite the Swedish law on record keeping (52), this was something that was rarely documented by the healthcare professionals.

The healthcare professionals also highlighted the importance of a team-based approach. Teamwork was shown to reduce constipation among older patients when staff participated in a quality improvement method (53). However, Klein and Holowaty (54) found no reduction in constipation after the implementation of a Constipation Management Protocol, although the healthcare professionals became more observant of the patients' normal bowel pattern and documentation improved. Lack of guidelines might be related to inadequate documentation, which has also been found in palliative care (55). Lee (56) argues that recordkeeping is important and in Sweden this is also mandatory by law. When preventive care processes, such as Senior Alert, have been implemented this has resulted in better structure and quality of care (57). Thus, the implementation of constipation risk assessment in the quality improvement register Senior Alert should be encouraged. When guidelines are used for best practice, there 
needs to be clearly identified clinical management and active communication between all members of the team (58) as well as effective team functioning (59).

All the participants were recruited from one medium-sized hospital with geriatric wards which may be a limitation. However, the participants' represented a substantial variation in age, gender and work experience. The strength of this study was the use of a combination of focus groups and individual interviews, since rich data were provided about how healthcare professionals manage problems related to constipation. Another strength is that different professional perspectives were represented in relation to experiences of assessment, treatment and prevention, which may be valuable when implementing strategies and improving the care of older patients.

\section{Conclusions}

In this study, healthcare professionals described constipation as being an important issue. Different strategies and person-centered care were considered necessary as was the ability for clinical reasoning; however, they found this to be difficult. It is important that healthcare professionals can reflect on their own knowledge and clinical practice. This will ensure that patients receive treatments that are more individualized rather than standardized. Overall, the results underscore the importance of adequate access to resources and education in constipation management and that clinical guidelines could be a guide for delivering high quality care in hospitals.

\section{Declarations}

\section{Ethics approval and consent to participate}

Ethical approval was provided for the project by the Regional Board of Research Ethics in Stockholm (2016/2314-31/4). In accordance with the ethical guidelines of the Helsinki declaration (60), all participants received oral and written information about the study and its purpose and signed informed consent before data collection took place. We advised the participants that they could withdraw from participating without giving a reason.

\section{Consent for publication}

Not applicable

\section{Availability of data and materials}

The datasets analysed during the current study are not available. According to the ethical approval, only the members of the research team are granted access to these data sets.

\section{Competing interests}

The authors declare that they have no competing interest. 


\section{Funding}

This study was funded by a grant from the Swedish Foundation Stockholms Sjukhem and internal grants from division of nursing at Karolinska Institutet. The findings, conclusions or recommendations expressed in this publication are those of the authors.

\section{Author contributions}

Study design: VL, HK; data collection: VL, HK; data analysis: VL, HK and manuscript preparation: VL, HK, $A M B, K G$.

\section{Acknowledgement}

The authors thank all the nurses and physicians for their participation and for sharing their valuable experiences.

\section{References}

1. Suares NC, Ford AC. Prevalence of, and risk factors for, chronic idiopathic constipation in the community: systematic review and meta-analysis. Am J Gastroenterol. 2011; doi:10.1038/ajg.2011.164

2. Camilleri M, Ford AC, Mawe GM, Dinning PG, Rao SS, Chey WD, et al. Chronic constipation. Nat Rev Dis primers. 2017; DOI: 10.1038/nrdp.2017.95

3. Lamas K, Karlsson S, Nolen A, Lovheim H, Sandman PO. Prevalence of constipation among persons living in institutional geriatric-care settings - a cross-sectional study. Scand j caring sci. 2017; DOI: $10.1111 /$ scs. 12345

4. Schmidt FM, Santos VL. Prevalence of constipation in the general adult population: an integrative review. J Wound, Ostomy Continence Nurs. 2014; DOI: 10.1097/01.WON.0000438019.21229.b7

5. Potter J, Wagg A. Management of bowel problems in older people: an update. Clin Med (London). 2005; DOI: 10.7861/clinmedicine.5-3-289

6. Johanson JF, Kralstein J. Chronic constipation: a survey of the patient perspective. Aliment Pharmacol Ther. 2007; DOI: 10.1111/j.1365-2036.2006.03238.x

7. Gallegos-Orozco JF, Foxx-Orenstein AE, Sterler SM, Stoa JM. Chronic constipation in the elderly. Am J Gastroenterol. 2012; DOI: 10.1038/ajg.2011.349

8. Dimidi E, Dibley L, Cotterill N, Scott M, Whelan K, Knowles CH, et al. Validated constipation symptom and quality-of-life measures neither reflect patient and clinician concerns nor use words familiar to patients. Gastrointestinal Nursing. 2016; Doi:10.12968/gasn.2016.14.8.29

9. Leung L, Riutta T, Kotecha J, Rosser W. Chronic constipation: an evidence-based review. J Am Board Fam Med. 2011; Doi:10.3122/jabfm.2011.04.100272 
10. Mearin F, Lacy BE, Chang L, Chey WD, Lembo AJ, Simren M, et al. Bowel Disorders. Gastroenterology. 2016;Doi:10.1053/j.gastro.2016.02.031

11. Gallagher P, O'Mahony D. Constipation in old age. Best Pract Res Clin gastroenterol. 2009; Doi: 10.1016/j.bpg.2009.09.001

12. Higgins PD, Johanson JF. Epidemiology of constipation in North America: a systematic review. Am J Gastroenterol. 2004; Doi:10.1111/j.1572-0241.2004.04114.x

13. Leonard R, Tinetti ME, Allore HG, Drickamer MA. Potentially modifiable resident characteristics that are associated with physical or verbal aggression among nursing home residents with dementia. Arch Intern Med. 2006; Doi:10.1001/archinte.166.12.1295

14. Munch L, Tvistholm N, Trosborg I, Konradsen H. Living with constipation-older people's experiences and strategies with constipation before and during hospitalization. Int J Qual Stud Health Well-being. 2016; Doi:10.3402/qhw.v11.30732

15. Tvistholm N, Munch L, Danielsen AK. Constipation is casting a shadow over everyday life - a systematic review on older people's experience of living with constipation. J Clin Nurs. 2017; Doi:10.3402/qhw.v11.30732

16. Hasson F, Muldrew D, Carduff E, Finucane A, Graham-Wisener L, Larkin P, et al. 'Take more laxatives was their answer to everything': A qualitative exploration of the patient, carer and healthcare professional experience of constipation in specialist palliative care. Palliat Med. 2019; Doi:10.1177/0269216319891584

17. Holroyd S. How can community nurses manage chronic constipation? JCN. 2015;29:74-82.

18. Rey E, Balboa A, Mearin F. Chronic constipation, irritable bowel syndrome with constipation and constipation with pain/discomfort: similarities and differences. Am J Gastroenterol. 2014; Doi:10.1038/ajg.2014.18

19. Dibley L, Coggrave M, McClurg D, Woodward S, Norton C. "It's just horrible": a qualitative study of patients' and carers' experiences of bowel dysfunction in multiple sclerosis. J Neurol. 2017; Doi:10.1007/s00415-017-8527-7

20. Chokhavatia S, John ES, Bridgeman MB, Dixit D. Constipation in Elderly Patients with Noncancer Pain: Focus on Opioid-Induced Constipation. Drugs Aging. 2016; Doi:10.1007/s40266-016-0381-2

21. Toner F, Claros E. Preventing, assessing, and managing constipation in older adults. Nursing. 2012; Doi:10.1097/01.NURSE.0000422642.83383.17

22. Woolery M, Bisanz A, Lyons HF, Gaido L, Yenulevich M, Fulton S, et al. Putting evidence into practice: evidence-based interventions for the prevention and management of constipation in patients with 
cancer. Clin J Oncol Nurs. 2008; Doi: DOI: 10.1188/08.CJON.317-337

23. Spinzi G, Amato A, Imperiali G, Lenoci N, Mandelli G, Paggi S, et al. Constipation in the elderly: management strategies. Drugs Aging. 2009; Doi:10.2165/00002512-200926060-00003

24. Senior Alert. Senior Alert - a national quality register for the preventive care of older people (Senior Alert - ett nationellt kvalitetsregister för vård och omsorg). 2020.

HTTP://plus.rjl.se/senioralert.Accessed 1 March 2020.

25. Larkin PJ, Sykes NP, Centeno C, Ellershaw JE, Elsner F, Eugene B, et al. The management of constipation in palliative care: clinical practice recommendations. Palliat Med. 2008; Doi:10.1177/0269216308096908

26. Polit DF, Beck CT. Nursing research: generating and assessing evidence for nursing practice. 10 th ed. Philadelphia: Wolters Kluwer; 2020.

27. Patton MQ. Qualitative research \& evaluation methods. 3rd ed. London: SAGE Publications; 2002.

28. Barbour R. Qualitative Research kit: Doing Focus Groups. London: SAGE Publications; 2007.

29. Graneheim UH, Lindgren BM, Lundman B. Methodological challenges in qualitative content analysis: A discussion paper. Nurs Educ Today. 2017; DOI: 10.1016/j.nedt.2017.06.002

30. Graneheim UH, Lundman B. Qualitative content analysis in nursing research: concepts, procedures and measures to achieve trustworthiness. Nurs Educ Today. 2004; Doi:10.1016/j.nedt.2003.10.001

31. Elo S, Kyngäs H. The qualitative content analysis process. J Adv Nurs. 2008; DOI: 10.1111/j.13652648.2007.04569.x

32. Holloway I, Galvin K. Qualitative Research in Nursing and Healthcare. $4^{\text {th }}$ ed. West Sussex: John Wiley \& Sons, Incorporated; 2016.

33. Elo S, Kääriäinen M, Kanste O, Pölkki T, Utriainen K, Kyngäs H. Qualitative content analysis: A focus on trustworthiness. SAGE open. 2014; DOI:10.1177/2158244014522633.

34. Saga S, Seim A, Morkved S, Norton C, Vinsnes AG. Bowel problem management among nursing home residents: a mixed methods study. BMC Nurs. 2014; DOI: 10.1186/s12912-014-0035-9

35. Richmond JP, Devlin R. Nurses' knowledge of prevention and management of constipation. Br J Nurs (Mark Allen Publishing). 2003; DOI: 10.12968/bjon.2003.12.10.11302

36. Zhe $\mathrm{H}$. The assessment and management of constipation among patients with advanced cancer in a palliative care ward in China: a best practice implementation project. JBI Database System Rev Implement Rep. 2016; DOI: 10.11124/JBISRIR-2016-002631

37. Izumi K. The measures to evaluate constipation: a review article. Gastroenterol Nurs. 2014; DOI: 10.1097/SGA.0000000000000034

38. Grainger M, Castledine G, Wood N, Dilley C. Researching the management of constipation in longterm care. Part 2. Br J Nurs. 2007; DOI: 10.12968/bjon.2007.16.18.27506 
39. Woolley N. Nursing diagnosis: exploring the factors which may influence the reasoning process. $J$ Adv Nurs. 1990;15(1):110-7. DOI: 10.1111/j.1365-2648.1990.tb01679.x

40. Muller-Staub M, de Graaf-Waar H, Paans W. An Internationally Consented Standard for Nursing Process-Clinical Decision Support Systems in Electronic Health Records. Comput Inform Nurs. 2016; DOI: $10.1097 / C I N .0000000000000277$

41. Harris LA, Horn J, Kissous-Hunt M, Magnus L, Quigley EMM. The Better Understanding and Recognition of the Disconnects, Experiences, and Needs of Patients with Chronic Idiopathic Constipation (BURDEN-CIC) Study: Results of an Online Questionnaire. Adv Ther. 2017; DOI: 10.1007/s12325-018-0733-x

42. Emmanuel A, Mattace-Raso F, Neri MC, Petersen KU, Rey E, Rogers J. Constipation in older people: A consensus statement. Int J Clin Pract. 2017; DOI: 10.1111/ijcp.12920

43. Trads M, Deutch SR, Pedersen PU. Supporting patients in reducing postoperative constipation: fundamental nursing care - a quasi-experimental study. Scand J Caring Sci. 2018; DOI: $10.1111 /$ scs. 12513

44. McCormack B, McCance TV. Development of a framework for person-centred nursing. J Adv Nurs. 2006; DOI: 10.1111/j.1365-2648.2006.04042.x

45. Warren D, Kent B. Determining the impact of a bowel management protocol on patients and clinicians' compliance in cardiac intensive care: A mixed-methods approach. J Clin Nurs. 2019; DOI: $10.1111 /$ jocn. 14669

46. Day MR, Wills T, Coffey A. Constipation and the pros and cons of laxatives for older adults. Nurs Residential Care. 2014; DOI: 10.12968/nrec.2014.16.4.196

47. Dimidi E, Cox C, Grant R, Scott SM, Whelan K. Perceptions of Constipation Among the General Public and People With Constipation Differ Strikingly From Those of General and Specialist Doctors and the Rome IV Criteria. Am J Gastroenterol. 2019; DOI: 10.14309/ajg.0000000000000267

48. Smith S. Evidence-based management of constipation in the oncology patient. Eur J Oncol Nurs. 2001; DOI: 10.1054/ejon.2000.0119

49. Tavares CN, Kimbrel JM, Protus BM, Grauer PA. Petroleum jelly (vaseline balls) for the treatment of constipation: a survey of hospice and palliative care practitioners. Am J Hosp Palliat Care. 2014; 10.1177/1049909113502578

50. Andresen V, Banerji V, Hall G, Lass A, Emmanuel AV. The patient burden of opioid-induced constipation: New insights from a large, multinational survey in five European countries. United European gastroenterol J. 2018; DOI: 10.1177/2050640618786145

51. Goodman M, Low J, Wilkinson S. Constipation management in palliative care: a survey of practices in the United kingdom. J Pain Symptom Manage. 2005; DOI: 10.1016/j.jpainsymman.2004.06.013

52. The Swedish Parliament (Sveriges Riksdag). The Patient Record act (Patientjournallag) (1985:562) 2020. HTTPS://www.riksdagen.se/sv/dokument-lagar/dokument/svenskforfattningssamling/patientjournallag-1985562_sfs-1985-562. Accessed 1 March 2020. 
53. Osuafor CN, Enduluri SL, Travers E, Bennett AM, Deveney E, Ali S, et al. Preventing and managing constipation in older inpatients. Int J Health Care Qual Assur. 2018; DOI: 10.1108/IJHCQA-05-20170082

54. Klein J, Holowaty S. Managing constipation: implementing a protocol in a geriatric rehabilitation setting. J Gerontol Nurs. 2014; DOI: 10.3928/00989134-20140501-01

55. Wee B, Adams A, Thompson K, Percival F, Burslem K, Jobanputra M. How much does it cost a specialist palliative care unit to manage constipation in patients receiving opioid therapy? J Pain Symptom Manage. 2010; DOI: 10.1016/j.jpainsymman.2009.09.007

56. Lee A. Identifying and managing constipation in older people. Nurs Residential Care. 2016; DOI: 10.12968/nrec.2016.18.7.366.

57. Lannering C, Ernsth Bravell M, Johansson L. Prevention of falls, malnutrition and pressure ulcers among older persons - nursing staff's experiences of a structured preventive care process. Health Soc Care Community. 2017; DOI: 10.1111/hsc. 12400

58. Mcllfatrick S, Muldrew DHL, Beck E, Carduff E, Clarke M, Finucane A, et al. Examining constipation assessment and management of patients with advanced cancer receiving specialist palliative care: a multi-site retrospective case note review of clinical practice. BMC Palliat Care. 2019; 10.1186/s12904-019-0436-3

59. Borglin G, Hew Thach E, Jeppsson M, Sjogren Forss K. Registered nurse's experiences of continence care for older people: A qualitative descriptive study. Int J Older People Nurs. 2019; DOI: 10.1111/opn.12275

60. World Medical Association. World Medical Association Declaration of Helsinki: Ethical Principles for Medical Research Involving Human Subjects. JAMA. 2013; DOI: doi: 10.1001/jama.2013.281053.

\section{Tables}

Table 1 Characteristics of the study participants $(\mathrm{N}=20)$

\begin{tabular}{|c|c|c|c|}
\hline Characteristics & & $\mathrm{n}(\%)$ & Mean (range) \\
\hline Age (years) & & & $40.7(25-59)$ \\
\hline Gender & $\begin{array}{l}\text { Female } \\
\text { Male }\end{array}$ & $\begin{array}{ll}17 & (85) \\
3 & (15) \\
\end{array}$ & \\
\hline Profession & $\begin{array}{l}\mathrm{RN \dagger} \\
\mathrm{ANP} \\
\mathrm{MD} \S\end{array}$ & $\begin{array}{r}14(70) \\
1(5) \\
5 \quad(25)\end{array}$ & \\
\hline Care Experience (years) & & & $13.8(1.5-30)$ \\
\hline
\end{tabular}

$\dagger=$ Registered Nurse

$\ddagger=$ Advanced Nurse Practitioner

$\S=$ Medical Doctor

Page 17/18 
Table 2 Examples of the condensation and analysis process

\begin{tabular}{|c|c|c|c|c|}
\hline Meaning unit & Condensed meaning unit & Code & Sub-category & Category \\
\hline $\begin{array}{l}\text { that's how it is sometimes in health care, you want to do more, but } \\
\text { there is not enough time }\end{array}$ & $\begin{array}{l}\text { You wish you could do more, } \\
\text { but there is not enough time }\end{array}$ & $\begin{array}{l}\text { Lack of } \\
\text { time }\end{array}$ & $\begin{array}{l}\text { Depending on } \\
\text { organizational } \\
\text { resources }\end{array}$ & $\begin{array}{l}\text { Depending } \\
\text { on } \\
\text { resources }\end{array}$ \\
\hline $\begin{array}{l}\text { You have to count the number of days too, what is reasonable and } \\
\text { what is not reasonable to have bowel movements, has the patient } \\
\text { eaten less previous days, then they may not be able to go to the toilet } \\
\text { so often }\end{array}$ & $\begin{array}{l}\text { Have to count the number of } \\
\text { days what is reasonable and } \\
\text { not to have bowel } \\
\text { movements }\end{array}$ & $\begin{array}{l}\text { lount } \\
\text { the } \\
\text { number } \\
\text { of days }\end{array}$ & $\begin{array}{l}\text { Individualized } \\
\text { strategies }\end{array}$ & $\begin{array}{l}\text { Using } \\
\text { different } \\
\text { strategies }\end{array}$ \\
\hline
\end{tabular}

